\title{
ANÁLISIS DE LOS FACTORES QUE INFLUYEN EN LA SELECCIÓN DE UNA UNIVERSIDAD EN BACHILLERES DE LA CIUDAD DE GUAYAQUIL
}

\section{ANALYSIS OF THE FACTORS THAT INFLUENCE IN THE SELECTION OF A UNIVERSITY IN HIGH SCHOOL OF THE CITY OF GUAYAQUIL}

\author{
Jessica Cárdenas-García ${ }^{1}$, Greta Pinos-Bravo²
}

\begin{abstract}
Resumen
El presente trabajo tiene como objetivo analizar los factores que influyen en la selección de una universidad en un grupo de bachilleres de Guayaquil, basado en su imagen, prestigio, infraestructura y demás aspectos que, para los estudiantes, proyectan un valor agregado. En la revisión de literatura se intentó conceptualizar y explicar la relación de las variables de investigación establecidas como: imagen corporativa, calidad académica y la decisión de compra del consumidor. A través de esta investigación se diseñó una escala de medición basada en el estudio de García y Moreno (2012) referente a una lista de 10 autores que recopiló al menos 38 atributos recomendables para la selección de una universidad. En este caso, la escala de medición fue construida por un total de 34 ítems agrupados en 5 dimensiones como: factores de infraestructura, económico, académico, administrativos y sociales y prestaciones, con el fin de realizar una encuesta a un grupo de 196 bachilleres recién graduados de la ciudad de Guayaquil. Los resultados determinaron que la dimensión académica es la más influyente al momento de escoger una universidad, dado que tiene que ver con el prestigio de los docentes, los valores inculcados, la certificación de entes reguladores e inclusive la flexibilidad de horarios y la duración de los programas universitarios para que los estudiantes puedan estudiar y a la vez costear su programa académico.
\end{abstract}

Códigos JEL: A22

\footnotetext{
${ }^{1}$ ESAI Business School, Universidad Espíritu Santo, (Ecuador).

E-mail: jacardenas@uees.edu.ec

${ }^{2}$ ESAI Business School, Universidad Espíritu Santo, (Ecuador).

E-mail: gpinos@uees.edu.ec
} 
Keywords:

Academy, Corporate Image, Prestige

\begin{abstract}
The present work aims to analyze the factors that influence the selection of a university in a group of high school graduates from Guayaquil, based on its image, prestige, infrastructure and other aspects that, for students, project added value. In the literature review, an attempt was made to conceptualize and explain the relationship of the established research variables such as: corporate image, academic quality and the consumer's purchase decision. Through this research, a measurement scale was designed based on the study by García and Moreno (2012) referring to a list of 10 authors who collected at least 29 recommended attributes for the selection of a university. In this case, the measurement scale was constructed by a total of 34 items grouped into 5 dimensions such as: infrastructure, economic, academic, administrative and social factors and benefits, in order to conduct a survey of a group of 196 recent high school graduates. graduates of the city of Guayaquil. The results determined that the academic dimension is the most influential when choosing a university, since it has to do with the prestige of the teachers, the values instilled, the certification of regulatory bodies and even the flexibility of schedules and the duration of the courses. university programs so that students can study and at the same time pay for their academic program.
\end{abstract}

\section{INTRODUCCIÓN}

La educación superior ha atravesado un proceso de cambio permanente que busca alinear el conocimiento con las nuevas tecnologías de la información y comunicación (TIC), complementándose en el proceso de enseñanza aprendizaje, en busca de lograr una educación tecnológica de vanguardia basada en el conocimiento real y actualizado (Garcés et al., 2016).

En el Ecuador, el ente regulador de la educación superior es la Secretaría Nacional de Ciencia y Tecnología (SENESCYT), pero a este sistema se sumaron otras instituciones como el Consejo de Educación Superior (CES) y el Consejo de Aseguramiento de la Calidad de Educación Superior (CACES), cuyos objetivos se fundamentan en servir de semillero para el impulso de una investigación científica, principalmente, en proyectos de innovación considerados como pilares para el desarrollo de sectores estratégicos en el país, a fin de consolidar una sociedad del conocimiento (Molina et al., 2020).
Esta situación trajo consigo diversos requisitos que las universidades debían cumplir para ser consideradas como aptas para garantizar una educación superior de calidad; sistema al que se sujetaron todas las universidades del país (Guzmán, 2011); ya sean públicas, particulares que reciben rentas del Estado y particulares autofinanciadas. Esta acreditación generó que algunas universidades cerrarán sus puertas porque no cumplían con los requisitos que avalen la calidad académica, mientras que otras fueron ganando mayor prestigio porque, además de contar con una infraestructura óptima, disponían de un personal docente profesional y comprometido en la formación de nuevos profesionales dentro del marco académico, ético y moral que se exige en el mundo laboral (Benito, 2017).

En los últimos 30 años, surgieron diversas universidades privadas en el país, motivadas por los ideales de un grupo de académicos que querían hacer una universidad diferente, entre las cuales apostaron al desarrollo de competencias basadas en el idioma extranjero (Benito, 2017); así como 
también el uso de plataformas digitales para lograr una mayor interacción entre el estudiante, los docentes y el personal administrativo (García, 2017). Todo esto, junto con una amplia oferta académica de pregrado y posgrado, trajo consigo un crecimiento del número de estudiantes a lo largo de todo este tiempo, logrando que varias de estas nuevas universidades se posicionen como una de las mejores del país, recibiendo la acreditación del CACES (Consejo de Aseguramiento de la Calidad de la Educación Superior, 2019).

Debido a que seleccionar una universidad es una de las decisiones más importantes en la vida de toda persona que desee iniciar una carrera profesional (Figueroa, 1993), este tema ha sido objeto de estudio por muchos académicos que han abordado su problemática desde varios enfoques. Dentro de estos debates se mencionan los pro y contras de incluir ciertos factores en el análisis, a causa de la variedad y complejidad de medición de algunas dimensiones (Bravo \& Vergara, 2018).

En gran medida, estos debates se generan por el hecho de incluir, dentro el análisis, diversos factores que garanticen la satisfacción de los estudiantes por la universidad y la carrera que escogen (García \& Moreno, 2012). Esto se debe a que, cuando una persona ingresa por primera vez a la universidad, no puede evaluar su calidad y sentirse completamente satisfecho hasta que culmine sus estudios

Sin embargo, su selección inicial estará basada en la percepción que éstos tienen sobre otras personas de su entorno más cercano, o de las acreditaciones que tenga dicha institución para servir de soporte o garantía de la calidad académica que el estudiante busca (Briones \& Triviño, 2018). Por esta razón, el objetivo general de esta investigación es determinar los factores que influyen en la selección de una universidad en un grupo de bachilleres de Guayaquil, para lo cual se estudian aspectos como: imagen, prestigio, infraestructura, y factores afines que para los estudiantes proyectan un valor agregado.

En este contexto, el estudio se justifica porque, hoy en día, las exigencias de los clientes por bienes y servicios que no sólo sean de calidad, sino que además satisfagan sus necesidades y les aporte valor, es una situación que determina que las organizaciones requieran estudios que analicen la percepción del cliente (Paride, 2017). $\mathrm{Al}$ referirse a la percepción, las empresas intentan descifrar la manera en que son vistos por el mercado, según la imagen y prestigio que proyectan hacia la comunidad (Mora Contreras, 2011). Por esta razón, con la información que se obtengan de estos estudios es posible diseñar estrategias que faciliten una interacción más directa entre el cliente y la empresa, en este caso, las universidades y sus estudiantes (García \& Moreno, 2012).

Veloutsou et al. (2005) argumentan que algunos estudiantes escogen una universidad basado en los atributos o factores que les proyectan, y no se apoyan tanto en las consejerías de orientación vocacional. Por otro lado, Weicheng (2003) explica que la omisión de factores sociales y culturales en el análisis puede traer conflictos internos en el estudiante, dado que el tema de la integración social es un aspecto que también es vital al momento de escoger una universidad.

Diversos autores concuerdan en el hecho de que, si desde un inicio no se aborda este tema, puede generarse una problemática que se evidencia en las bajas relaciones sociales que tienen ciertas personas (De la Mano et al., 2013). Esta situación se manifiesta en aquellas instituciones académicas donde su selección también se forja con base en el vínculo social que pueden tener los estudiantes; siendo un aspecto vital al momento de entablar nuevos contactos para desarrollar sociedades y nuevos negocios en el futuro (Montesano \& Zambrano, 2013).

De esta forma, al hacer una revisión de literatura sobre los atributos que debe considerarse en el proceso de selección de una universidad, se aprecia que no hay un consenso entre los diversos autores, para determinar con precisión los mejores factores, ya que como se mencionó, estos pueden variar según la percepción que tenga el estudiante sobre una institución y de la necesidad que realmente desea satisfacer. 
Por tal razón, a través de esta investigación se pretende diseñar una escala de medición que ayude a evaluar aquellos factores o atributos que consideran los bachilleres de la ciudad de Guayaquil, al momento de elegir una universidad y qué importancia tienen estos factores. Por tanto, se trata de un estudio de enfoque cuantitativo para que a través de una encuesta se obtengan datos más precisos sobre la percepción que los bachilleres tienen sobre la imagen, prestigio o infraestructura de una entidad académica, a fin de diagnosticar lo que realmente los motiva en su decisión de selección final.

\section{REVISIÓN DE LITERATURA}

\section{Imagen Corporativa de las Instituciones de Educación Superior}

En la actualidad la educación ha revolucionado las diferentes esferas sociales, porque su impulso hacia el desarrollo y la calidad ha sido una solicitud de estado para de esta manera generar calidad en el sector (D'Armas et al., 2018). Por medio de la era tecnológica la educación ha despuntado una variedad de ofertas atractivas a fin de que los interesados no dejen sus estudios por razones de tiempo o distancia, el crédito y las becas han premiado la responsabilidad y dedicación de estudiantes de escasos recursos económicos que desean superarse (Takaki et al., 2015).

Es así como las diferentes modalidades online, semipresencial y a distancia han tomado posición en el sector para que los estudiantes puedan adaptar los horarios a sus necesidades (Dos Santos, 2016). Por este motivo las universidades tratan diversificar sus propuestas y proyectar una imagen corporativa de prestigio $y$ calidad.

Toda entidad con o sin fines de lucro necesita proyectar una imagen corporativa que genere confianza y fortaleza en el sector donde se desarrolla la actividad comercial o el servicio, por este motivo las Instituciones de Educación Superior tienen que ser competitivas para mantenerse operativas y atraer el interés de profesionales que puedan compartir la misma visión a los estudiantes (Navarro, 2003), finalmente estos son la mejor fuente de publicidad, ya que por medio de las recomendaciones y de su perfil profesional generarán en el público interés por la institución de educación superior (Montero-Mora \& CantónCroda, 2020)

Según estudios el trabajo de marca en instituciones educativas se realiza de forma poco recurrente, volviéndose difícil de examinar los motivos que validen la elección entre una Institución Educativa y otra, por la falta de información que este tipo de estudio tiene (Basurto \& Flores, 2016)

Bajo este contexto es necesario proyectar la identidad que posee toda institución y posteriormente gestionarla y promoverla como imagen corporativa a través de los diferentes canales de información para de esta manera tener contacto con el público y conocer sus intereses, necesidades y lo que esperan de la oferta, para posteriormente canalizar esta información de acuerdo con lo que el estudiante espera recibir (Hernández-Gil et al., 2018).

De esta manera se reconoce la excelencia de los estudiantes que poseen calificaciones meritorias a través de las becas, las cuales son cada vez más reconocidas, por esta razón las instituciones hacen atractivos los títulos y resaltan su infraestructura a fin de que la demanda académica los reconozca como una entidad de élite (Martínez, 2011).

La buena imagen de una institución no está relacionada únicamente con la marca, sino que esta es apreciada por el cliente a través de aquellos aspectos que más recuerdan de la entidad (Terán, 2019). En este sentido es importante analizar lo que piensa el público perceptor y clientes o estudiantes de la universidad, para así determinar el impacto que tiene la gestión de la imagen a través de diferentes acciones que determinan el reconocimiento de la institución y sus motivos (Blanco, 2018).

El proceso para dar una identidad a un negocio está desarrollado en 4 partes, como lo revela Molina et al. (2020) (a) identidad visual;
(b)
comunicaciones;
(c) cultura $\mathrm{y}$ 
comportamiento, este patrón es tomado en cuenta para la elaboración de propuestas de identidad corporativa en el marco estudiantil. Sin embargo, para efectos de este trabajo se tomará como referencia la selección de factores que realizaron García y Moreno (2012) referente a una lista de diez autores con publicaciones recientes, donde se distinguen al menos 29 atributos que son recomendables de utilizar para evaluar la decisión de selección de una universidad:

Tabla 1.

Principales atributos que se utilizan para evaluar la decisión de selección de una universidad

\begin{tabular}{|c|c|c|}
\hline $\begin{array}{l}\text { - Costo } \\
\text { - Ubicación } \\
\text { - Infraestructura } \\
\text { - Privada/pública } \\
\text { - Requisitos de } \\
\text { admisión } \\
\text { - Pruebas requeridas } \\
\text { - Distancia } \\
\text { - Servicios o } \\
\text { programas } \\
\text { extracurriculares } \\
\text { - Vida social al } \\
\text { interior de la } \\
\text { universidad } \\
\text { - Nivel académico }\end{array}$ & $\begin{array}{l}\text { - Reputación de la } \\
\text { institución } \\
\text { - Oportunidades de } \\
\text { empleo } \\
\text { - Consolidación de } \\
\text { la carrera } \\
\text { - Tipo y duración } \\
\text { de los programas } \\
\text { académicos } \\
\text { - Amigos y } \\
\text { familiares } \\
\text { - Experiencia de } \\
\text { los docentes } \\
\text { - Fomento y apoyo } \\
\text { a deportes } \\
\text { - Tamaño de la } \\
\text { universidad } \\
\text { - Número de } \\
\text { estudiantes por } \\
\text { maestro } \\
\text { - Enfoque de la } \\
\text { carrera }\end{array}$ & 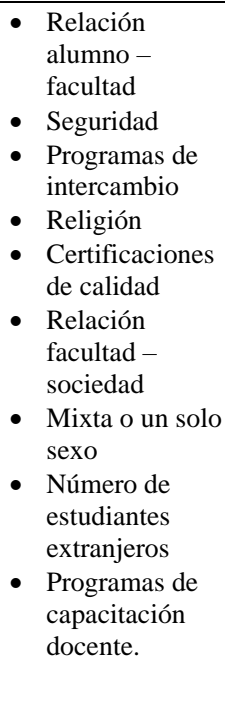 \\
\hline
\end{tabular}

\section{Decisión en la elección y contratación de servicios}

La importancia y necesidad de dar a conocer una institución educativa para atraer más clientes, es necesaria para generar una incidencia de compra o adquisición, estudiar la manera en la que se debe abordar esta gestión es vital para obtener resultados positivos. Granados y Alameda (2017) detallan que el cliente determina cuando y a quien comprar cuando encuentra aquello que satisface sus necesidades y lo expresa en la siguiente frase: "un montón de frentes y todos en guerra" (p. 114).

La marca debe mantener una conexión con el cliente por medio de razones que influencian en la decisión de compra del consumidor, en este caso del estudiante, debido a que actualmente el medio de interacción no es convencional, ofreciendo nuevos retos el mundo digital es el mecanismo actual que sirve de vitrina para dar a conocer un producto o servicio.

De acuerdo con García y Gastulo (2018) el cliente que necesita un producto o servicio no siempre busca satisfacerlo a cabalidad, sino que se deja llevar por la novedad, innovación y el reconocimiento, sin embargo esto a veces varía en función del costo, ya que cuando se trata de algo que tiene un valor adquisitivo relevante la decisión de compra es pensada y muchas veces consensuada en familia, por este motivo la resolución por parte del cliente en estudios de tercer y cuarto nivel va a estar ligada al factor: calidad/precio.

En este contexto Piqueras (2017) sostiene que el vínculo calidad - precio tiene que ver con la apreciación que el cliente tenga de que el servicio que recibe compensa el costo monetario de inversión, es decir que siempre va a existir una elevada expectativa con la calidad que estará relacionada al precio. De acuerdo con lo sostenido se puede inferir en que cuando existe un precio inferior el cliente considerará que la calidad también lo es y por el contrario cuando el precio es elevado se espera una mayor calidad.

Bajo este contexto se puede determinar que la decisión de compra estará sujeta a las expectativas del cliente relacionadas al factor calidad/precio, de acuerdo con Schiffman y Kanuk (2010) delimita el proceso de decisión de compra en 3 etapas: (1) el input o entrada, ese tiene que ver con la observación y exploración; (2) el proceso, momento en que el cliente realiza comparaciones o autoevalúa todos los aspectos que recibió en el proceso de entrada, en esta etapa se involucran aspectos psicológicos: estímulos de descuento, la percepción de calidad-precio, el aprendizaje y la personalidad; y (3) el output o la salida, es la etapa donde el cliente toma la decisión final, después de una rigurosa valoración.

En la segunda etapa se desarrolla un análisis de la información relacionada al producto, calidad y la experiencia de otro individuo o su recomendación, con la finalidad de valorar todos los criterios receptados y que por lo general se 
asocian a características como calidad, precio, entrega, ubicación, entre otros.

\section{La calidad académica como índice de prestigio}

Según Águila (2012) la demanda de trabajo en el continente americano se sujeta a la calidad de universitarios; que captan profesionales con un perfil académico relevante, donde el promedio es valorado al momento de escoger personal. Bajo esta afirmación se puede deducir que la competencia de profesionales se da por la búsqueda de calidad y excelencia, es así que estar preparados para un mundo competitivo es un reto que se forja en las aulas de clases. Por tal motivo la calidad debe surgir como eje de valores y culturalidad insertados en la misión de la institución universitaria quien es la responsable de ofrecer profesionales competitivos, con valores éticos y morales que traspasen las fronteras de la investigación y pensamiento.

En el contexto histórico la calidad se ha ido forjando a través de ciertos patrones que ofrece la concepción de estructuras flexibles que direccionan un proceso crítico de valoración; este desempeño que realiza la institución en su oferta académica está vinculada a la estructura social en la que realiza su actividad económica. La finalidad de una Universidad es ofrecer educación de calidad, para así suplir los requerimientos de los estudiantes y de los sectores productivos que necesitan profesionales competentes en las diferentes especialidades; la educación ofrece un abanico de oportunidades que son aprovechadas por las personas para gestar sus proyectos personales y de vida.

La creatividad juega un papel fundamental para desarrollar un posicionamiento a largo plazo, ya que actualmente vivimos en una era cambiante $\mathrm{y}$ para permanecer en el mercado y ser competitivo se deben aprovechar todas las oportunidades que surjan de las necesidades no suplidas por la competencia (Martínez, 2013). Innovar no solo es mejorar la calidad educativa, este aspecto abarca a la entidad de forma general, ya que todos sus departamentos poseen igual importancia, debido a que las instituciones que buscan la globalización de su oferta curricular deben analizar sus manuales de procesos, para que estos suplan las necesidades del consumidor final de forma eficiente y eficaz (Martínez, 2013).

\section{Relación entre imagen, calidad y decisión de compra}

El resultado de una imagen corporativa se evidencia en las acciones que tengan los consumidores, proveedores y toda la estructura de mercado (Johnson y Wilson, 1993; Helm, 2007). La fortaleza de una marca se demuestra con el compromiso de la empresa hacia su cliente a través de la calidad de sus productos (Vallester y de Chernatony, 2006), por este motivo es necesario gestionar beneficios comunes para que el cliente se sienta parte de la institución (Rooney, 1995; Andreassen y Lindestad, 1998).

Es relevante discernir el comportamiento del cliente, ya que no todo acercamiento positivo quiere decir que la empresa ha logrado su cometido, es decir la proyección de imagen corporativa, por tal motivo se debe realizar un trabajo cognitivo en el público para posteiormente identificar aspectos como satisfacción, compromiso y lealtad siendo estas las piezas claves para medir el impacto emocional de la marca en el cliente (Woodruff et al., 1991; Rust y Oliver, 1994). Por otro lado, hay quienes consideran que las experiencias del cliente dan como resultado la creación de la imagen corporativa (Cronin y Taylor, 1992; Rindell et al., 2010), y su complacencia será resultado de las expectativas alcanzadas (Kim et al., 2008; Hu et al., 2009).

Sharma et al. (2009) considera que la imagen corporativa es resultado de una situación conciliadora que vincula satisfacción y lealtad, es decir que cuando la imagen proyectada es fuerte, el vínculo de pertenencia también se consolida. El trabajo de González et al. (2009) sostiene que la imagen y satisfacción no se relacionan con la lealtad, ya que la satisfacción es resultado de la imagen y lo que percibe el cliente respecto a los trabajadores, instalaciones, estrategias y gestiones desarrolladas por la empresa van a contribuir en el nivel de complacencia que tengan los clientes con la entidad. 
Chaudhuri y Holbrook (2001, 2002) sostiene que la lealtad y compromiso son efecto de una imagen que satisface las necesidades del cliente y por consiguiente estos estarían dispuestos a ser fiel a la marca, bajo esta premisa la reputación o la percepción de habilidades y valores de la compañía son parte de la imagen proyectada, sin embargo las características de la marca como creatividad e ingenio, colorido, tamaño, forma van a ser reconocidos solo por aquellos clientes que sienten compromiso o se sienten identificados con la imagen corporativa de la empresa.

\section{METODOLOGÍA}

Con base en lo previamente expuesto, la investigación se desarrolló bajo un diseño no experimental, dado que no se manipularon las variables que forman parte del estudio (Hernández, Fernández, \& Baptista, 2015). Además, la investigación fue de alcance transversal porque los datos se recogieron en un momento único y específico, y no se analizaron varios períodos.

Para la toma de datos, la población objeto de estudio se identifica como el total de bachilleres recién graduados, quienes son postulantes en las diferentes universidades públicas y privadas del Ecuador. Los datos del Ministerio de Educación determinan que, en la región Costa, se graduaron un total de 152.354 estudiantes bachilleres de unidades educativas fiscales, fiscomisionales, municipales y particulares, durante el período 2020 - 2021. De los cuales se estimó que al menos la mitad corresponden a estudiantes de la provincia del Guayas, lo que da como resultado una población equivalente a 76.177 alumnos y, de este grupo el $70 \%$ pertenecen a Guayaquil, es decir que la población objeto de estudio corresponde a 53.323 bachilleres de Guayaquil.

Para efectos de esta investigación se aplicó un muestreo no probabilístico - por conveniencia, enfocado a un total de 160 alumnos, que sería la cantidad de bachilleres que formarían parte del estudio. Por esta razón, la muestra fue dividida en cuatro establecimientos de educación básica para para recoger sus opiniones sobre los factores que inciden en la selección de una universidad local. En este contexto, se pudo recoger 80 datos de dos instituciones particulares como: Thomas More, Ecomundo y 80 datos de dos entidades educativas públicas como: Colegio Guayaquil y Colegio Carlos Julio Arosemena Tola.

Además, se diseñó un instrumento de medición que, de forma efectiva, permitió evaluar los diferentes factores que influyen en la decisión final de un grupo de bachilleres de Guayaquil, al momento de escoger una universidad. Este instrumento se apoyó en una escala de medición con base en la investigación de García y Moreno (2012) quienes habían elaborado un cuestionario de 38 ítems divididos en cinco dimensiones como: infraestructura, económicos, académicos, administrativos y sociales y prestaciones. No obstante, se escogieron sólo 34 ítems para la elaboración de este cuestionario, dado que las 4 preguntas que se excluyeron no eran pertinentes para el contexto de estudio ecuatoriano. Además, para obtener la fiabilidad o consistencia del cuestionario se calculó el índice de alfa de Cronbach, el cual arrojó un valor de 0.930 por todos los ítems utilizados en esta escala.

Para el levantamiento de datos se utilizó como técnica una encuesta, dado que Gallardo (2017) argumenta que se trata de una técnica versátil para recolectar una gran cantidad de información, en poco tiempo, y su facilidad se manifiesta en que se apoya en un cuestionario de preguntas cerradas, es decir, que poseen una respuesta estandarizada, bajo un modelo, como la escala de Likert, donde las respuestas fueron valoradas entre 1 y 5 , donde 1 representa muy en desacuerdo y 5 muy de acuerdo.

Por efectos de la pandemia del covid-19, la toma de datos se hizo a través de un cuestionario digital por medio de la herramienta de Google Forms, que permitió difundir el cuestionario a una gran cantidad de personas en poco tiempo e hizo posible la visualización de los datos en tiempo real. Además, como unidad de análisis se consideró al número de estudiantes bachilleres que se graduaron en la región Costa hasta el período 2019 - 2020 .

Finalmente, la investigación fue descriptiva porque se hizo una caracterización de las dimensiones que involucran la percepción de 
calidad de la imagen corporativa, es decir, qué aspectos se desagregan de la variable calidad, y cómo eso influye en la decisión de elegir una universidad.

\section{RESULTADOS}

Para facilitar la interpretación de los resultados, el análisis de las 34 respuestas fue agrupado en las cinco dimensiones previamente establecidas. Además, los ítems inmersos dentro de cada dimensión fueron promediados para obtener un dato más fácil de interpretar. Por esta razón, las valoraciones de 1 al 3 se consideran como negativas porque determinan el menor nivel de conformidad de los encuestados; a diferencia de las valoraciones entre 4 y 5 que representan un mayor nivel de conformidad o importancia (García \& Moreno, 2012)

Al consultar sobre la dimensión de infraestructura, se pudo notar que el 60\% (suma de valores 4 y 5) consideró que este es un aspecto importante al momento de escoger una universidad, influenciado mayormente por aspectos como: bibliotecas, laboratorios especializados y centros de cómputo, por encima de aspectos como: las instalaciones deportivas o el tamaño mismo de la universidad. Mientras que para el $40 \%$ esta dimensión no es tan relevante, tal como se observa en la figura 1.

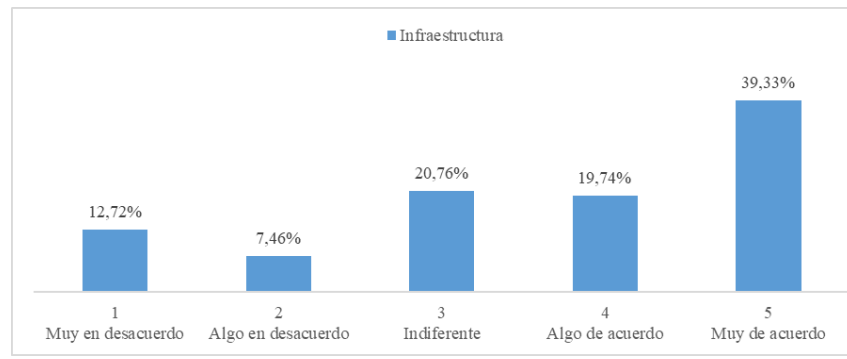

Figura 1. Evaluación de la dimensión Infraestructura

En la figura 2 se presentan los resultados de la dimensión académica, y aquí se aprecia que prácticamente todos los ítems obtuvieron una valoración de 5 con una participación del 44.15\%, siendo la nota más alta. En conjunto si se suman las notas 4 y 5 se obtiene que el $65 \%$ de encuestados valora más la dimensión académica.
Ante lo cual se interpreta que esta dimensión es posiblemente la más influyente en su decisión de compra de algún programa académico. Por tanto, para el bachiller es muy importante el prestigio académico, los valores inculcados por la universidad, el índice de egresados que están insertados en el campo laboral, el prestigio de los docentes, la flexibilidad de horarios y la duración de los períodos universitarios. Sin embargo, el ítem que tuvo una menor valoración dentro de esta dimensión fue el hecho de que la universidad promueva el intercambio estudiantil, por lo que se podría considerar que se trata de un factor poco relevante en la decisión de elegir una universidad.

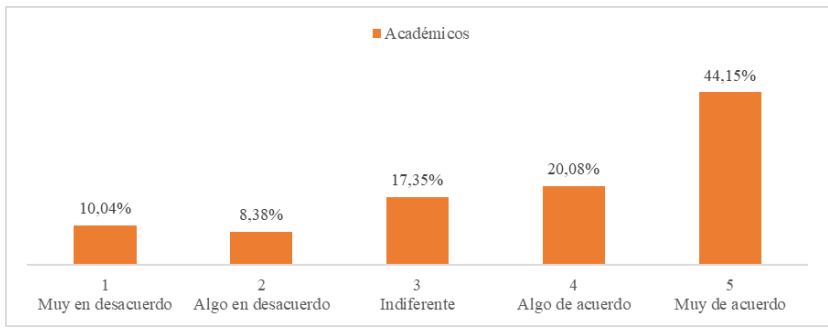

Figura 2. Evaluación de la dimensión Académica

Por otro lado, la dimensión administrativa también tuvo valoraciones entre 4 y 5 , siendo destacado el hecho de que es poco relevante para el bachiller que la universidad tenga convenios internacionales al momento de elegirla. Otros ítems como la doble titulación, la calificación mínima aprobatoria, o ágiles procesos de admisión también son poco relevantes para que el estudiante escoja una universidad, pues, este tipo de situaciones normalmente las experimentarán al momento de ser estudiantes una entidad académica, y mientras son postulantes, es difícil saber qué tan relevante eso puede ser para ellos.

No obstante, aspectos como el enfoque del programa teórico - práctico y el contenido de las asignaturas, fueron los ítems que se destacaron al presentar valoraciones entre 4 y 5 y concentrar casi el $70 \%$ de las opiniones. Lo que posiblemente establece que estos dos aspectos relacionados con la malla curricular y la calidad de contenidos sí podría ser influyente al momento de elegir una universidad, tal como se observa en la figura 3. 


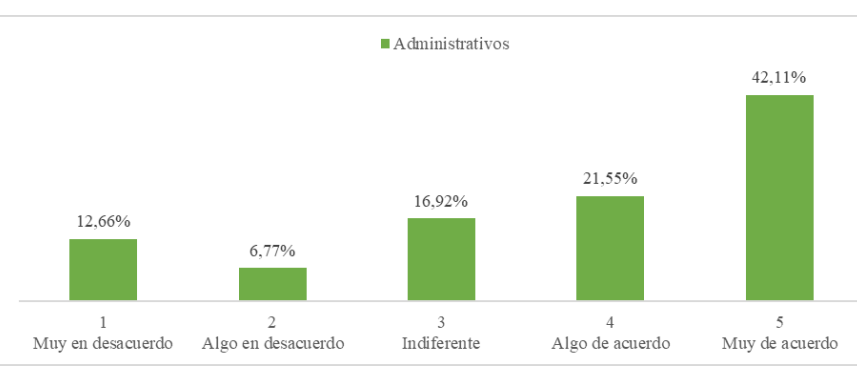

Figura 3. Evaluación de la dimensión administrativa

De acuerdo con la evidencia empírica, el factor económico a veces suele ser influyente al momento de adquirir un bien o servicio; sin embargo, cuando se trata de la educación superior, el precio no determina necesariamente una decisión de compra, pues se trata de un servicio de alta implicancia y es algo que marcará la vida de una persona, porque tiene que ver con su formación como profesional.

Por esta razón, los resultados determinaron que en ciertos aspectos como el hecho de que sea una universidad privada o pública es poco relevante, incluyendo el costo de la matrícula y la posibilidad de obtener una beca. Es decir, los estudiantes estarían dispuesto a pagar un valor alto si a cambio recibe una formación académica de calidad, pero también un factor determinante es que los programas educativos ofrezcan la posibilidad de trabajar al mismo tiempo, a fin de poder costear el valor de la carrera, ante lo cual sí no hay horarios flexibles posiblemente sí se vea afectada su decisión de compra.

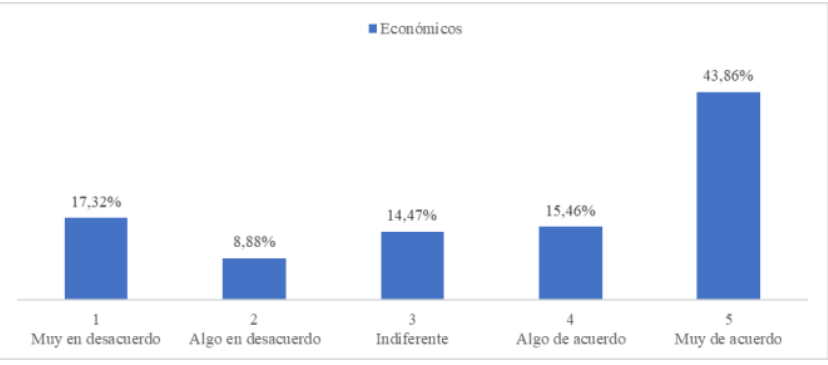

Figura 4. Evaluación de la dimensión económica

Finalmente, como parte del instrumento de medición aplicado fue pertinente consultar acerca de la influencia que tienen los factores sociales y las prestaciones dentro de la decisión de elegir una universidad. Los resultados determinan que, para el estudiante es poco relevante el entorno cotidiano de convivencia, las actividades extracurriculares y que sus amigos estudien ahí, pues si bien puede ser un factor importante, pasa a segundo plano cuando el prestigio y calidad académica quizás determine que no toda persona esté apta para entrar a un programa universitario, ante lo cual el tema social no es tan influyente.

Por esta razón, esta dimensión concentró el $47 \%$ de opiniones entre notas 4 y 5 , mientras que el $53 \%$ valoró entre 1 y 3 . Sin embargo, dada la situación de pandemia del Covid-19, algunos bachilleres mencionaron que un factor clave dentro de este punto sería que la universidad sí disponga de prestaciones de servicio de salud, para solventar alguna emergencia que se pudiera suscitar durante la jornada de clases.

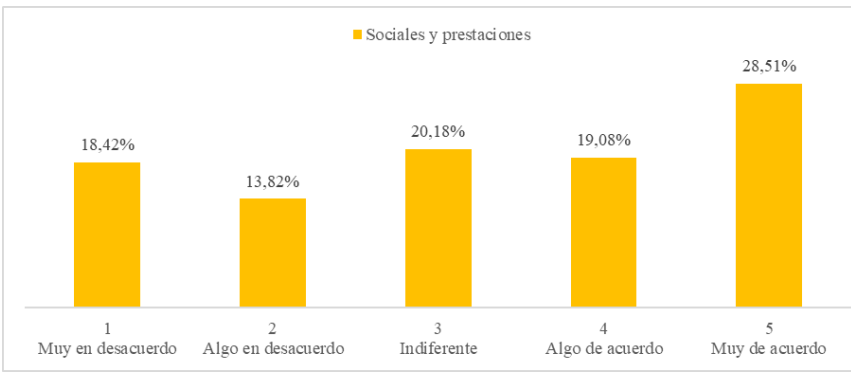

Figura 5. Evaluación de la dimensión sociales y prestaciones

En este sentido, los datos obtenidos determinan que prácticamente todas estas dimensiones son altamente influyentes para el estudiante, pero definitivamente sobresalen 2 elementos claves como factores decisivos al momento de escoger una universidad, estos son: la parte académica y la parte administrativa. Esto puede ser evidenciado en la tabla 2 que presenta un análisis de correlación de estas cinco dimensiones, las cuales fueron abreviadas de la siguiente forma: INF por infraestructura, ACA por académico, ADM por administrativo, ECO por económico y SOC por sociales y prestaciones.

De acuerdo con el análisis correlacional, todos los elementos tienen una fuerte correlación directa, lo que se interpreta al mostrar valores positivos. Sin embargo, es notorio destacar que las variables que tuvieron una correlación muy cercana a uno (1) fueron las dimensiones 
académicas y administrativas. Por tanto, se puede concluir que estos dos elementos son los más decisivos a la hora de seleccionar una universidad, inclusive por encima del factor económico, la infraestructura y los aspectos sociales y las prestaciones del campus.

Tabla 2.

\section{Análisis de Correlación}

\begin{tabular}{lccccc}
\hline & $I N F$ & $A C A$ & $A D M$ & $E C O$ & SOC \\
\hline INF & 1.0000 & & & & \\
ACA & 0.9838 & 1.0000 & & & \\
ADM & 0.9860 & $\mathbf{0 . 9 9 1 5}$ & 1.0000 & & \\
ECO & 0.9312 & 0.9484 & 0.9512 & 1.0000 & \\
SOC & 0.9835 & 0.9541 & 0.9692 & 0.9540 & 1.0000 \\
\hline
\end{tabular}

\section{CONCLUSIONES}

Una vez realizada la investigación, se puede concluir que al momento de analizar los factores que influyen en la selección de una universidad en un grupo de bachilleres de Guayaquil, a través de un análisis de correlación, se establece que la imagen y prestigio de una institución de educación superior se define en función de los aspectos académicos y administrativos que proyecte a los postulantes. En este caso, estas dos dimensiones fueron las que mayor peso tuvieron dentro de las elecciones de los 160 bachilleres que formaron parte del muestreo, tanto de entidades educativas particulares como del sector público.

Un aspecto interesante fue notar que el factor económico no se convierte en una variable relevante al momento de escoger una universidad, lo que determina que los jóvenes están conscientes de que su formación académica se trata de una inversión que posteriormente será recuperada al momento de acceder a plazas de trabajo que le permitan poner en práctica todos esos conocimientos adquiridos. Por tanto, el factor académico impulsa ese aspecto de competitividad en los estudiantes porque le da mayor seguridad de poder desenvolverse mejor en el mercado laboral.

\section{Fortalezas y Limitaciones}

En torno a esta investigación, la principal fortaleza fue contar con el apoyo de un grupo de 160 bachilleres de unidades educativas públicas y particulares, quienes formaron parte del muestreo y vertieron sus opiniones para el desarrollo de este trabajo. Sin embargo, una limitación es el tamaño de la muestra, dado que por el factor tiempo y las condiciones de pandemia no se pudo hacer una encuesta a un mayor número de bachilleres, ni tampoco se pudo tener un contacto físico con estas personas, ante lo cual no se pudo dialogar con ellos para interpretar de una forma más profunda sus respuestas.

\section{Futuras Líneas de Investigación}

Finalmente, se recomienda que se desarrollen más estudios que apliquen un instrumento de medición basado en determinar los factores más determinantes al momento de elegir algún bien o servicio. Si bien este trabajo se enfocó en el campo de educación superior, esta escala podría ser replicada y adaptada para el sector empresarial, a fin de conocer los motivos por los cuales los productos de una compañía son escogidos o valorados por sus clientes; aspecto que determinaría la necesidad de mantener o mejorar la calidad del bien o servicio ofertado.

\section{REFERENCIAS}

Basurto, K., \& Flores, O. (2016). Imagen institucional de la Universidad de Montemorelos percibida por los maestros y personal de apoyo. Research Gate, 1(1), 1-61. https://doi.org/10.13140/RG.2.2.15313.63842

Benito, V. (2017). Las Políticas Públicas de Educación en Ecuador, como una de las manifestaciones e instrumentos del Plan Nacional para el Buen Vivir. Universidad de Alicante, 1(1), $1-789$.

Blanco, T. (2018). Españolas, La Construcción de Imagen Corporativa en las Universidades. Revista Internacional de Ciencias Sociales y Humanidades SOCIOTAM, 28(2), 1-11. 
Bravo, G., \& Vergara, M. (2018). Factores que determinan la elección de carrera profesional: en estudiantes de undécimo grado de colegios públicos y privados de Barrancabermeja. Psicoespacios, 12(20), 35-48. https://doi.org/10.25057/21452776.1000

Briones, Y., \& Triviño, J. (2018). Factores que intervienen para elegir carreras universitarias. Universidad Técnica de Manabí. Adaya Press., 1(1), 200-207. http://www.adayapress.com/wpcontent/uploads/2018/04/20.pdf

Consejo de Aseguramiento de la Calidad de la Educación Superior. (2019). Modelo de Evaluación Externa de Universidades y Escuelas Politécnicas. https://www.caces.gob.ec/wpcontent/uploads/downloads/2019/12/3.-

Modelo_Eval_UEP_2019_compressed.pdf

D’Armas, M., Robles, R., Cedillo, M., Vinueza, J., Correa, M., Mejías, A., \& Acosta, G. (2018). Imagen institucional universitaria: Un estudio de caso en el contexto del Ecuador. Espacios, 39(5), $1-16$.

De la Mano, M., Moro, M., \& Da Graca, M. (2013). Motivaciones en la elección de la carrera universitaria. EDICI, 1(1), 1-18.

Dos Santos, M. (2016). Calidad y satisfacción : el caso de la Universidad de Jaén. Revista de La Educacion Superior, 45(178), 79-95. http://www.scielo.org.mx/pdf/resu/v45n178/018 5-2760-resu-45-178-00079.pdf

Figueroa, E. (1993). La elección de carrera: una decisión de gran trascendencia. Educación, 11(3), 5-13.

Garcés, E., Garcés, E., \& Alcívar, F. (2016). Las tecnologías de la información en el cambio de la educación en el siglo XXI. Reflexiones para la práctica. Universidad y Sociedad, 8(4), 171-177.

García, J., \& Moreno, C. (2012). Factores considerados al seleccionar una universidad: Caso Ciudad Juárez. Revista Mexicana de Investigacion Educativa, 17(52), 287-305.
García, S. (2017). Alfabetización Digital. Razón y Palabra, 21(98), 66-81. http://www.redalyc.org/pdf/1995/199553113006. pdf

García, T., \& Gastulo, D. (2018). Factores que influyen en la decisión de compra del consumidor para la marca Metro - Chiclayo. Universidad Católica Santo Toribio de Mogrovejo, 68. http://tesis.usat.edu.pe/bitstream/20.500.12423/1 039/1/TL_GarciaGrandaTatianaLisseth_Gastulo ChuzonDoritaNatali.pdf.pdf

Granados, P., \& Alameda, D. (2017). El proceso de decisión de compra: canales online y canales tradicionales. Investigación y Marketing, 113(12), 29-37.

Guzmán, J. (2011). La calidad de la enseñanza en la Educación Superior ¿Qué es una buena enseñanza en este nivel educativo? Perfiles Educativos, 33(1), 129-141. https://doi.org/10.23857/dc.v5i3.934

Hernández-Gil, C., Figueroa-Ramírez, E. F., \& Correa-Corrales, L. E. (2018). Reposicionamiento de marca: el camino hacia la competitividad de las pequeñas y medianas empresas. Revista De Investigación, Desarrollo E Innovación, 9(1), 33-46. https://doi.org/10.19053/20278306.v9.n1.2018.8 505

Martínez, F. (2011). Los rankings de universidades: una visión crítica. Revista de La Educación Superior, 1(157), 77-97.

Molina, T. D. J., Burbano, L. H., Lizcano, C. J., \& Viteri, J. M. (2020). Evaluación y acreditación de las Universidades en Ecuador. Caso: Universidad Regional Autónoma de Los Andes. Educación, $41 \quad$ (20)(20), 297-309. https://www.revistaespacios.com

Montero-Mora, J. G., \& Cantón-Croda, R. M. (2020). Validación de un instrumento para medir satisfacción de usuarios en instituciones educativas del sector privado: ciudad de XalapaEnríquez (México). Innovaciones Educativas, 22(32), 122-136. https://doi.org/10.22458/ie.v22i32.2727 
Montesano, J., \& Zambrano, E. (2013). Factores que influyen en la elección de una carrera universitaria en la Universidad Católica Andrés Bello.

http://biblioteca2.ucab.edu.ve/anexos/biblioteca/ marc/texto/AAS6028.pdf

Mora Contreras, C. E. (2011). La Calidad del Servicio y la Satisfacción del Consumidor. Revista Brasileira de Marketing, 10(2), 146-162. https://doi.org/10.5585/remark.v10i2.2212

Navarro, E. R. (2003). El rendimiento académico: concepto, investigación y desarrollo. REICE. Revista Iberoamericana Sobre Calidad, Eficacia y Cambio En Educación, 1(2), 1-16. http://www.ice.deusto.es/rinace/reice/vol1n2/Ede 1.pdf

Paride, B. (2017). Satisfacción del Cliente. Thema, 10. http://actualidadempresa.com/satisfaccion-delcliente-importancia-e-infraestructura-necesaria/

Piqueras, C. (2017). La íntima relación entre calidad y precio. https://www.cesarpiqueras.com/relacion-entrecalidad-y-precio/\#: :text=La relación calidadprecio $\% 2 \mathrm{C}$ es,eso se espera de ella.

Schiffman, L., \& Kanuk, L. (2010). Comportamiento del Consumidor. Prentice Hall México.

Takaki, M., Bravo, R., \& Martínez, E. (2015). La gestión de la identidad corporativa en la Universidad: análisis y consecuencias desde la perspectiva del profesorado. Revista Europea de Direccion y Economia de La Empresa, 24(1), 2534. https://doi.org/10.1016/j.redee.2014.05.001

Terán, J. L. (2019). El branding corporativo como estrategia en el rediseño de marca de la agencia CREA SION Publicidad del Cantón Daule, Parroquia La Aurora. Universidad de Guayaquil, 1(1), 1-102.

Veloutsou, C., Paton, R. A., \& Lewis, J. (2005). Consultation and reliability of information sources pertaining to university selection: some questions answered? International Journal of Educational Management, 19(4), 279-291.

Weicheng, Z. (2003). Bridging cultural diff erences in college selection and application. The Journal of College Admission, 180, 6-17. 


\section{APÉNDICES \\ FORMATO DE ENCUESTA}

Dirigido a: Bachilleres de Guayaquil

Estimado encuestado, favor llenar el presente formulario cuyo objetivo es analizar los factores que influyen en la selección de una universidad en un grupo de bachilleres de Guayaquil, basado en su imagen, prestigio, infraestructura y demás aspectos que, para los estudiantes, proyectan un valor agregado. Tomar en consideración las siguientes instrucciones:

Responder con sinceridad, dado que el cuestionario es anónimo.

Leer bien cada pregunta y escoger una respuesta por cada ítem o pregunta.

La valoración se establece entre 1 y 5 , interpretándose a 1 como la respuesta de mayor desacuerdo o poco

relevante, y 5 la respuesta como muy relevante.

Está de acuerdo con que su respuesta sea utilizada para fines académicos:

Sí_- No_

\begin{tabular}{|c|c|c|c|c|c|c|}
\hline \multirow[b]{2}{*}{ No. } & Variables y Dimensiones & \multirow[b]{2}{*}{1} & \multirow[b]{2}{*}{2} & \multirow[b]{2}{*}{3} & \multirow[b]{2}{*}{4} & \multirow[b]{2}{*}{5} \\
\hline & $\begin{array}{l}\text { INFRAESTRUCTURA: Se refiere a todas las edificaciones físicas y áreas que tienen las } \\
\text { universidades como bibliotecas, laboratorios, canchas, aulas y demás espacios que } \\
\text { capten su interés. }\end{array}$ & & & & & \\
\hline 1 & $\begin{array}{l}\text { La Universidad de su interés cuenta con bibliotecas propias en sus facultades o al menos } \\
\text { una general. }\end{array}$ & & & & & \\
\hline 2 & Existen laboratorios especializados para la carrera de su interés. & & & & & \\
\hline 3 & Las aulas son adecuadas para la realización de prácticas. & & & & & \\
\hline 4 & Cuenta con centros de cómputo aptos para los estudiantes en cualquier consulta. & & & & & \\
\hline 5 & Existen instalaciones para realizar actividades deportivas & & & & & \\
\hline \multirow[t]{2}{*}{6} & El tamaño de la universidad capta su interés & & & & & \\
\hline & $\begin{array}{l}\text { ACADÉMICOS: Se enfoca en la parte educativa, en el prestigio académico que } \\
\text { transmiten sus docentes o profesionales graduados. }\end{array}$ & & & & & \\
\hline 7 & La Universidad tiene prestigio académico & & & & & \\
\hline 8 & La Universidad le proyecta valores inculcados en la escuela & & & & & \\
\hline 9 & Los índices de egresados con trabajo son altos con relación a otras universidades. & & & & & \\
\hline 10 & Le llama la atención los cursos de aprendizaje de otros idiomas & & & & & \\
\hline 11 & Escoge la universidad por el prestigio de los docentes & & & & & \\
\hline 12 & La Universidad cuenta con una certificación de calidad por los organismos rectores & & & & & \\
\hline 13 & Le interesa que la universidad promueva el intercambio estudiantil & & & & & \\
\hline 14 & Capta su interés que la universidad tenga flexibilidad en los horarios de clases & & & & & \\
\hline \multirow[t]{2}{*}{15} & Es importante la duración y forma de los períodos escolares & & & & & \\
\hline & $\begin{array}{l}\text { ADMINISTRATIVOS: Tiene que ver con aquellos aspectos que regulan los programas } \\
\text { de estudio, horarios, modalidades de titulación, convenios, admisiones, malla curricular, } \\
\text { etc. }\end{array}$ & & & & & \\
\hline 16 & Le llama la atención que la universidad tenga convenios internacionales & & & & & \\
\hline 17 & Capta su interés el hecho de que el enfoque del programa sea teórico o práctico. & & & & & \\
\hline 18 & Es importante que le ofrezca varias opciones de titulación & & & & & \\
\hline 19 & Es importante para Ud. que las carreras ofrezcan doble titulación & & & & & \\
\hline 20 & Influye en su decisión la calificación mínima aprobatoria & & & & & \\
\hline 21 & El proceso de admisión tiene que ser ágil & & & & & \\
\hline 22 & Le importa mucho el contenido de las asignaturas & & & & & \\
\hline
\end{tabular}




\begin{tabular}{|l|l|l|l|l|} 
& \multicolumn{1}{|l|}{} & & & \\
\hline & $\begin{array}{l}\text { ECONÓMICOS: Se relaciona con el costo que implica invertir en un programa } \\
\text { académico, para determinar si es un factor influyente en la elección de una universidad. }\end{array}$ & & & \\
\hline 23 & Le interesa mucho que la universidad sea pública & & & \\
\hline 24 & Le interesa mucho que la universidad sea privada & & & \\
\hline 25 & Un factor importante es el costo de la matrícula & & & \\
\hline 26 & Influye en su decisión el costo de los materiales didácticos y libros que debe comprar & & & \\
\hline 27 & Es importante para ud la facilidad de obtener una beca & & & \\
\hline 28 & Influye mucho en su decisión el monto de la beca & & & \\
\hline 29 & Le interesa la posibilidad de estudiar y trabajar simultáneamente & & & \\
\hline 30 & El valor de la titulación es influyente & & & \\
\hline & & & & \\
\hline & $\begin{array}{l}\text { SOCIALES Y PRESTACIONES: tiene que ver con las relaciones que se generan con } \\
\text { otros estudiantes dentro de la universidad. }\end{array}$ & & & \\
\hline 31 & El entorno cotidiano como la convivencia entre alumnos es influyente & & & \\
\hline 32 & La interesa que existan actividades extracurriculares & & \\
\hline 33 & Escoge la universidad porque ahí estudian sus amigos & & \\
\hline 34 & Escoge la universidad porque tiene prestaciones de servicios de salud & & & \\
\hline
\end{tabular}

